DOI: $10.21892 / 978-958-5547-65-0.2$

\title{
2. Los incentivos como política para favorecer las innovaciones ambientales
}

\author{
Alexandra Cumbe-Figueroa ${ }^{1}$
}

\section{Introducción}

Los incentivos ambientales surgen a partir de la necesidad Estatal de adoptar medidas eficientes y eficaces para la protección del ambiente, que se está viendo gravemente afectado por las actividades de sobreexplotación de recursos naturales, sobre la cual se encuentra fundada el modelo económico de la superproducción, que lo ha llevado al punto de afectar sus procesos naturales de renovación (Espinoza, 2001).

Esto representa grandes consecuencias para la supervivencia de las especies que hacen parte de la naturaleza, y afectan de manera directa la salud, la vida y la integridad física de los seres humanos, por lo que, los Estados y Organizaciones mundiales como la ONU, han dedicado esfuerzos en los últimos años para fijar medidas internacionales tendientes a proteger, conservar y recuperar el ambiente a través de tratados y convenios internacionales (Cruz, Gallego \& González, 2009).

Colombia ha ratificado y aprobado un gran número de estos instrumentos jurídicos internacionales, adquiriendo obligaciones en materia ambiental, entre ellos, está el Convenio de Brasilia de 1992 sobre la Diversidad Biológica, en el que se dispuso la obligación de los Estados, de adoptar, en la medida de lo posible, medidas económicas o sociales como incentivos para la conservación y la utilización de los recursos naturales dentro del marco del desarrollo sostenible (Organización de Naciones Unidas, 1992).

Con base a esto, es que Colombia ha venido incluyendo en su legislación, incentivos ambientales de diferentes tipos, a través de los cuales pretende que aquellos que realizan actividades contaminantes o las van a realizar, procuren mitigar los daños ambientales, o en su defecto no

1 Estudiante de Derecho de la Universidad La Gran Colombia. 
los produzcan por el incentivo económico o social que pueden recibir a cambio. Estas medidas han permitido concebir otras formas de utilización de los recursos naturales, aplicado el concepto de desarrollo sostenible, dando cuenta que la aplicación del de medidas normativas que están a la vanguardia pueden contribuir a la conservación y recuperación del ambiente.

Para contribuir con esta fundamentación de los incentivos ambientales como medidas eficientes para proteger el ambiente, nos aproximaremos a la contextualización de la afectación ambiental. Posteriormente, se revisarán las obligaciones internacionales de Colombia en materia ambiental y, finalmente, se presentaran algunos de los incentivos ambientales que ha incluido Colombia como una política pública para cumplir tanto con los fines del Estado como con los compromisos internacionales.

\section{Afectación ambiental: contextualización}

El gran avance de la sociedad en los últimos 40 años es innegable, pese a las desigualdades las condiciones de vida de la sociedad han mejorado, ha aumentado la esperanza de vida y han mejorado las comunicaciones, aunque de manera paralela el hombre ha explotado los recursos naturales de una manera desenfrenada, al punto de llegar a afectar la naturaleza lo suficiente como para impedirle que desarrolle su proceso de renovación, lo que es una amenaza para el ambiente propiamente dicho y para la supervivencia de todas las especies que en él habitamos (Espinoza, 2001).

Los problemas ambientales, encuentran su principal origen en los factores socioeconómicos y políticos, inevitablemente unidos a la sobreexplotación de los recursos naturales, tanto de las formas de apropiación como de distribución, de las relaciones económicas del mundo, de la soberanía interna sobre la naturaleza y de la relocalización de las industrias, más que en los límites que la tierra tiene para apoyar el desarrollo del ser humano (Amaya, 2002).

Si bien los recursos naturales fueron, por mucho tiempo, los instrumentos de un desarrollo económico que no establecía muchas restricciones, hoy por hoy, los cambios que han dejado estos avances económicos, han permeado en los ámbitos culturales y políticos del mundo 
moderno, tanto así, que los países de manera individual no pueden superar los conflictos ambientales como el calentamiento global de la atmosfera, el daño a la capa de ozono, el cambio climático, la pérdida de biodiversidad y reducción de las vegetaciones (Espinoza, 2001).

Es en razón a ello que, la problemática ambiental se convierte en el centro de la atención política: (i) por la magnitud de las repercusiones ambientales de local a mundial; $y$, (ii) porque, de acuerdo con los ecologistas políticos, las aproximaciones monotemáticas que pretenden enfrentar los problemas ambientales resultan insuficientes. (Amaya, 2002).

Sus primeros acercamientos, se dan en la década de los 70, donde los problemas ambientales aumentaban notablemente producto del desarrollo económico. Lo que lleva a que comiencen los Estados a tomar medidas, y a avanzar en la cooperación internacional para la conservación y la recuperación de los recursos naturales. Así se puede ver, con el aumento en el número de tratados y acuerdos internacionales que, en este sentido, se han promulgado para la protección ambiental (Cruz, Gallego \& González, 2009).

De estos esfuerzos universales encaminados a la protección del ambiente, nacen nuevos conceptos que buscan la armonía entre la conservación de la naturaleza y el desarrollo de la humanidad, entre ellos, el desarrollo sostenible, desde el cual deja de percibirse como la satisfacción de las necesidades del presente, para pasar a pensar en las generaciones venideras, procurando por el crecimiento, estabilidad y modernización del ser humano en un ambiente estable y sano (Espinoza, 2001).

De manera que, esta explotación desmedida de los recursos naturales para lograr el desarrollo económico, tras las grandes afectaciones generadas al ambiente, éste encuentra límite en el desarrollo sostenible que propende por el desarrollo económico por medio de la explotación contralada de los recursos naturales, teniendo respeto por los procesos naturales del mismo y su necesidad de renovarse.

2. Obligaciones internacionales de Colombia en materia ambiental

Como se ha mencionado, la explotación desmesurada de los recursos naturales y la consecuente degradación ambiental se ha convertido, en la actualidad, en una preocupación universal, que se ve reflejada en el gran número de tratados y convenios internacionales expedidos sobre la 
materia (Caballero, 1997). Frente a esto, el hecho de que Colombia ocupe el segundo lugar entre los doce países con mayor diversidad biológica del mundo, después de Brasil (Mittermeier \& Goettsch, 1997), le genera grandes responsabilidades al Estado tanto en el ámbito interno como en el internacional (Caballero, 1997)

En efecto, la agenda del Derecho internacional a partir del dinamismo que lo caracteriza, ha incluido desde la década de los 90 temas de la problemática ambiental. No obstante, la comunidad internacional ya había adelantado esfuerzos expidiendo algunas disposiciones en materia ambiental, que Colombia ratificó, entre ellas, la Declaración de Estocolmo sobre el Medio Ambiente Humano se ubica como el primer escenario mundial en el que se reunieron 113 Estados para considerar la conexión del ambiente con las necesidades específicas de desarrollo de los países, allí fijaron un conjunto de principios fundamentales sobre el ambiente, consagrando: el ambiente como derecho humano fundamental; el concepto de desarrollo sostenible; el principio de precaución, ante la incertidumbre del peligro del daño; la visión antropocéntrica, desde la cual se protege el ambiente para la no poder en peligro la existencia de la especie humana; la competencia a entidades locales ante eventos de afectación de los recursos naturales; y, el principio de racionalidad (González, 2006: Organización de Naciones Unidas, 1972).

Además de esto, la Declaración de Estocolmo tuvo como resultado la creación del Programa de las Naciones Unidas para el Ambiente- PNUMA, como organismo de la Organización de Naciones Unidas encargado de proporcionar liderazgo y promover los esfuerzos conjuntos para el cuidado con el ambiente en los Estados (Organización de Naciones Unidas, 1972)

Un año antes, se había adelantado la Convención sobre los Humedales de Ramsar en 1971, igualmente ratificada por Colombia y aprobada mediante la Ley 357 de 1997, que tenía por objeto expedir una serie de disposiciones sobre la conservación y uso racional de los humedales, por su importancia al equilibrio de los ecosistemas, como contribución al desarrollo sostenible de todo el mundo (Organización de las Naciones Unidas, 1971; Congreso de Colombia, 1997).

Asimismo, está el Tratado de Cooperación Amazónica de Brasilia de 1978, ratificado por Colombia y aprobado mediante la Ley 74 de 1979 , 
que tenía por objeto la cooperación de los países con regiones amazónicas en cuanto a estrategias de desarrollo integral en esta zona, buscando el equilibrio entre el crecimiento económico y la preservación del medio ambiente (Tratado de Cooperación Amazónica, 1978; Congreso de Colombia, 1975).

A continuación, en 1983, el Estado colombiano ratificó el Convenio de Cartagena para la protección y el desarrollo del medio marino en la región del Gran Caribe y el Protocolo de Cartagena sobre la cooperación para combatir los derrames de hidrocarburos en la región del gran caribe, ratificados ambos mediante la Ley 56 de 1987, estos instrumentos jurídicos se encargaron de establecer una serie de medidas encaminadas a la prevención de la contaminación en el ecosistema marino del Caribe y sobre todo, la generada por derrames de hidrocarburos (Organización de las Naciones Unidas, 1987; Congreso de la República, 1987).

Avanzando cronológicamente, en 1985 se expide el Convenio de Viena para la protección de la capa de ozono, que fue ratificado por Colombia y aprobado mediante la Ley 32 de 1985, este Convenio fue el resultado de la iniciativa global de reparar el daño causado a la capa de ozono por el uso de clorofluorocarbonos y otros productos químicos de la misma naturaleza; y se dispuso que los países firmantes se comprometían a investigar, compartir, informar y ejecutar medidas preventivas frente a la fabricación y emisiones de Sustancias Agotadoras de la Capa de Ozono -SAO- (Organización de las Naciones Unidas, 1985; Congreso de la República, 1985).

Posteriormente, se realizó el Convenio de Basilea de 1989 relativo al control de los movimientos transfronterizos de los desechos peligrosos y su eliminación, ratificado por Colombia y aprobado por la Ley 253 de 1996, en el que se estipularon una serie de disposiciones para controlar los movimientos transfronterizos de desechos peligrosos y la eliminación de estos en los territorios de cada Estado firmante (Organización de las Naciones Unidas, 1989; Congreso de la República, 1996).

De ahí, pasamos a la década de los 90, donde se realizó en 1992 la Conferencia de Río de Janeiro, donde se aprobaron 5 documentos: la Declaración de Principios, el Convenio sobre Biodiversidad, el Convenio sobre Cambio Climático, una Declaración sobre Boques y la Agenda de Trabajo. Fue la Declaración de Principios el instrumento encargado de 
reunir todos los principios que orientarían el Derecho ambiental, tomando los consagrados en la Conferencia de Estocolmo de 1972, entre ellos, se estableció la visión antropocéntrica, según la cual, no se protege a la naturaleza por sí misma, sino porque con la protección de los recursos naturales se logra garantizar la vida saludable y productiva de los seres humanos, reiterándose que el derecho a la salud y al ambiente son indisolubles (González, 2006; Organización de las Naciones Unidas, 1992).

Asimismo, esta Declaración establece las perturbaciones de vecindad, de acuerdo con la cual cada Estado es soberano para decidir sobre sus afectaciones hasta tanto no afecte el ambiente de otros Estados. También, se incluye el principio de precaución, ante la incertidumbre científica de la ocurrencia del daño al ambiente; la obligación de internalizar los costos ambientales; la obligación de hacer estudios de impacto ambiental a todo proyecto que pueda generar afectaciones ambientales, entre otros (Organización de las Naciones Unidas, 1992).

Por su parte, la Conferencia de Río también dejó como producto, la aprobación de la creación del Programa 21, como un acuerdo de las Naciones unidas para promover el desarrollo sostenible, en el cual participan tanto los Estados, como los individuos, grupos y organizaciones, en cuanto a la evaluación ambiental y en la toma de decisiones (Organización de las Naciones Unidas, 1992).

Dentro de la misma Conferencia de Río, se aprobó el Convenio sobre la Diversidad Biológica, aprobado por Colombia mediante la Ley 165 de 1994, como tratado internacional jurídicamente vinculante relativo a la conservación de la biodiversidad, el uso sostenible de sus componentes y la participación justa y equitativa de los beneficios resultantes de la utilización de los recursos genéticos (Organización de las Naciones Unidas, 1992; Congreso de la República, 1994).

Por otro lado, en 1994, se aprobó en París la Convención de las Naciones Unidas de lucha contra la Desertificación y la Sequía (UNCCD), que fue ratificada por Colombia mediante Ley 461 del 4 de agosto de 1998, en ella los Estados se comprometen a aplicar en las zonas afectadas por la desertificación y la sequía estrategias para la recuperación, conservación y aprovechamiento sostenible de los recursos terrestres e hídricos, para 
conseguir mejorar la calidad de vida de la población (Organización de las Naciones Unidas, 1994; Congreso de la República, 1998).

Después de esto, Colombia fue signataria del Protocolo de Kioto en 1997 relativa a la Convención marco de las Naciones Unidas sobre el cambio climático, aprobado mediante la Ley 629 del 2000, en el que se fijaron algunos parámetros para disminuir las emisiones de gases contaminantes (Organización de las Naciones Unidas, 1997; Congreso de la República, 2000).

Con la llegada del nuevo milenio, la Asamblea General de la Organización de Naciones Unidas aprobó la Resolución 55/2, en la cual fijaron los Objetivos de Desarrollo del Milenio, que iban a ser cumplidos para el año 2015 y a los cuales el Estado colombiano se comprometió a alcanzar, entre ellos, se trataron temas de "erradicar la pobreza; lograr la enseñanza de la primaria individual; promover la igualdad de géneros y la autonomía de la mujer; reducir la mortalidad infantil; mejorar la salud materna; combatir el VIH/SIDA, el paludismo y otras enfermedades; garantizar la sostenibilidad del medio ambiente; y fomentar la asociación mundial para el desarrollo" (Organización de las Naciones Unidas, 2000).

Pasando al 2002, se celebró la Cumbre Mundial sobre Desarrollo Sostenible en Johannesburgo que dio como resultado la Declaración de Johannesburgo sobre el Desarrollo Sostenible, en él se establecieron medidas tendientes al desarrollo sostenible, a la pobreza, a los derechos humanos y a la gestión de los recursos naturales, fijan un plan de acción para aplicar los compromisos asumidos por los Estados en la Cumbre de Río de 1992 (Organización de las Naciones Unidas, 2002).

Está también, el Convenio de Rotterdam sobre Consentimiento Informado Previo -PIC que entró en vigor en el año 2004, y que fue el producto de la Conferencia de Rotterdam realizada en 1998, representó un gran avance en cuanto a la garantía de la protección de los derechos de la población y del medio ambiente frente a los posibles peligros del comercio internacional de plaguicidas y productos químicos altamente peligrosos, esto es, la protección de la salud y del medio ambiente, este Convenio fue ratificado por Colombia y aprobado mediante la Ley 1159 de 2007.

Por último, está la XXI Conferencia sobre el Cambio Climático celebrada en París en el 2015, donde se negoció por un Acuerdo que establera 
medidas para la reducción de las emisiones de Gases de Efecto Invernadero (GEI), en el contexto del desarrollo sostenible, con ciertos objetivos concretos: (i) adelantar esfuerzos por limitar el aumento de la temperatura media mundial a $1,5^{\circ} \mathrm{C}$; (ii) aumentar la capacidad de adaptación a los efectos adversos al cambio climático, promover la asimilación al clima y procurar mantener bajas emisiones de Gases de Efecto Invernadero para no comprometer la producción de alimentos. Este Acuerdo tendría aplicabilidad desde el año 2015 hasta el año 2020 fecha en que termina la vigencia del Protocolo de Kioto.

Así las cosas, la expedición de instrumentos jurídicos internacionales permite ver la preocupación de la comunidad internacional, en cuanto a la protección, conservación y recuperación del ambiente, la cual se ha venido incrementando en los últimos años, por las consecuencias adversas que los conflictos ambientales producen tanto a los seres humanos, como a las demás especies que habitan el planeta Tierra.

\section{Los incentivos ambientales como política de Estado}

Revisado el concepto de afectación ambiental y los compromisos ambientales que ha adquirido el Estado colombiano en materia ambiental, debemos traer a colación el Convenio sobre la diversidad biológica de 1992, que dispuso el deber de cada Estado, de adoptar, en lo posible, medidas económicas y socialmente eficientes como incentivos para la conservación y utilización sostenible de los recursos naturales (Organización de las Naciones Unidas, 1992).

De acuerdo con Hecken y Bastiaensen (2010) los incentivos ambientales son mecanismos a través de los cuales se pretende estimular que las personas tengan actuaciones más responsables con el ambiente, y al mismo tiempo, son concebidos como mecanismos que armonizan los objetivos de los actores implicados y la protección ambiental. Estos incentivos ambientales, generalmente, son compensaciones económicas, por medio de las cuales, se busca que las personas que están afectando los recursos naturales, o los van a afectar, realicen acciones en beneficio del ambiente. 
Es en razón de este propósito y de la gran diversidad de actores y de conflictos que surgen frente a la utilización de los recursos naturales que existen diferentes tipos de incentivos además del económico, entre ellos se pueden identificar: (i) los institucionales, que contemplan la creación de reservas naturales, parques naturales nacionales y regionales, la promoción del ecoturismo, programas de eco-responsabilidad, y más; (ii) los reglamentarios, como aquellos de auditoria y monitorio de la biodiversidad, restricciones a la explotación de la tierra, derechos de explotación comunitaria; y (iii) culturales o sociales, relativos a los trabajos voluntarios para la conservación, programas de educación, etc.

Ahora bien, los incentivos ambientales, se diferencian de la regulación directa en que, esta última a través de un cuerpo normativo impone obligaciones o restricciones respecto al actuar de los individuos ante la utilización de recursos naturales, en cambio, los mecanismos de incentivos están encaminados a hacer que las personas o empresas adopten decisiones tendientes a proteger el ambiente, por los precios o información que enfrentan (Reyes \& Vargas, 2010).

Respecto a los incentivos económicos, debemos tener en cuenta que hay diferentes tipos, entre ellos, está el Pago por Servicios Ambientales PSA-, como una herramienta que consiste en la celebración de un acuerdo entre propietarios, poseedores, u ocupantes de buena fe y el interesado en la preservación y restauración de la zona en la que los primeros están asentados. En este acuerdo, se pacta una compensación que puede ser dineraria o en especie por adelantar actividades a la conservación o restauración del área y una condición de resultado, lo que permite: (i) generar alternativas económicas para la comunidad; y (ii) mejorar las condiciones de vida y bienestar de los beneficiarios (Corte Constitucional, Sentencia C 644 de 2017).

Por otra parte, están los impuestos y subsidios, que son un instrumento según el cual, se internalizan los costos de los daños ambientales, es decir, ante la existencia de un daño ambiental el causante debe pagar por esto, pues se tiene como propósito evitar que se ocasione dicho daño ambiental o que se mitigue en lo posible la producción del mismo. Pero, si en lugar de esto, no se produce ningún daño porque se disuadió al actor de realizar la actividad causal, puede haber un beneficio económico por ello (Levinson \& Shetty, 1992; Reyes \& Vargas, 2010). 
Así las cosas, los impuestos materializan el principio ambiental de quien contamina paga, contrario a los subsidios, que por motivo de la conservación de la naturaleza por parte de quien se abstiene a realizar las actividades causantes de daños ambientales, recibe a cambio beneficios, verbigracia, tributarios (Reyes \& Vargas, 2010).

Por último, se encuentran los permisos comercializables de desarrollo, que restringen el área en la que el propietario puede discrecionalmente decidir sobre el uso del suelo, y la autoridad fija el porcentaje sobre el área que puede ser utilizada por medio de permisos, este mecanismo funciona delimitando las áreas que pueden ser utilizadas y aquellas que deben ser conservadas (Reyes \& Vargas, 2010).

Bajo ese entendido, pasaremos a identificar algunos de los incentivos ambientales que consagra la legislación colombiana, tomando como punto de partida el Decreto 624 de 1989 por medio del cual se expide el Estatuto Tributario, que en su art. 57-2 adicionado por la ley 1450 de 2011, fija que los recursos que reciba el contribuyente y que tengan como destino el desarrollo de proyectos científicos, tecnológicos o de innovación, de acuerdo con los parámetros del Consejo Nacional de Beneficios Tributarios en Ciencia, Tecnología e Innovación no hacen parte de los ingresos constitutivos de renta o ganancia ocasional, asimismo, son ingresos no constitutivos de renta la remuneración de las personas naturales por la ejecución directa de labores científicas, tecnológicas o de innovación siempre que la remuneración haga parte de los recursos destinados para el proyecto.

En el mismo sentido, el art. 126-5 adicionado por la Ley 1536 de 2012 establece que las personas que realicen donaciones a la Unidad Administrativa Especial del Sistema de Parques Nacionales Naturales para apadrinar parques naturales y conservar bosques naturales tienen derecho a la deducción del 30\% del impuesto de renta sobre las donaciones efectuadas durante el periodo gravable. Paralelamente, el art. 158-1 modificado por la Ley 1819 de 2016 dispone que habrá deducción de impuesto de renta del periodo en el que se realicen donaciones e inversiones en investigación, desarrollo tecnológico e innovación, siempre que se cumplan las condiciones fijadas por el Consejo Nacional de Política Económica y Social 
También se fija en el art. 159 de este articulado modificado por la Ley 1819 de 2016 que, habrá deducción a impuestos cuando se invierta en la evaluación y exploración de recursos naturales no renovables.

Del mismo modo, el art. 207-2 adicionado por la Ley 788 de 2002 analizado en conjunto con el art. 235-2 adicionado por la Ley 1819 de 2016 con establecen que habrá rentas exentas sobre servicios de ecoturismo certificados; ventas de energía eléctrica generada por energía eólica, biomasa o residuos agrícolas, solar, geotérmica o de los mares, realizada únicamente por empresas generadoras, siempre que: (i) se tenga en cuenta en Protocolo de Kioto en cuanto al trámite, obtención y venta de certificado de emisión de bióxido de carbono; (ii) que por lo menos el 50\% de los recursos obtenidos por la venta de los certificados se inviertan en obras de beneficio social en la región donde opera el generador; y (iii) prestar el servicio de transporte fluvial con embarcaciones y planchones de bajo calado.

El Estatuto Tributario, incluye a su vez, los descuentos tributarios, así el art. 255 modificado por la Ley 1819 de 2016 determina que cuando una persona jurídica realiza de manera directa inversiones en control, conservación y mejoramiento del medio ambiente, tiene el derecho a un descuento del 25\% de las inversiones realizadas en el respectivo año gravable sobre la renta. Y el art. 255 modificado igualmente por la Ley 1819 de 2016, establece que las personas que inviertan en proyectos calificados por el Consejo Nacional de Beneficios Tributarios en Ciencia y Tecnología en Innovación como de investigación, desarrollo tecnológico o innovación, tienen derecho a descontar de su impuesto sobre la renta correspondiente el 25\% del valor invertido en estos proyectos durante el periodo gravable.

Finalmente, se señalará del Estatuto tributario en este escrito dos artículos relevantes: el art. 424, modificado por la Ley 1819 de 2016, que establece la lista de bienes que están excluidos del impuesto, y su venta o importación no genera impuesto, entre ellos, determinados productos agrícolas y animales de consumo humano; ciertos medicamentos; máquinas para uso agropecuario; artículos y aparatos de ortopedia; abonos minerales y químicos; insecticidas; raticidas; desinfectantes; sistemas de riego; equipos de transfusión de sangre; los dispositivos anticonceptivos femeninos; los computadores de escritorio o portátiles que no excedan de 50 UVT (Unidad Valor Tributario); los dispositivos móviles inteligentes que no excedan de 22 UVT; los alimentos de consumo humano y animal importados de países 
vecinos a los departamentos de Vichada, Guajira, Guainía y Vaupés para su consumo local en los mismos departamentos; maquinaria y equipos que generen y certifiquen reducciones de Gases de Efecto Invernadero - GEI destinados a proyectos o actividades registradas en Registro Nacional de Reducción de Emisiones de Gases Efecto Invernadero, y más.

En segundo lugar, está el art. 428 sobre importaciones que no causan impuestos, entre las cuales el literal F, adicionado por la Ley 223 de 1995, fija la importación de maquinaria o equipos que no sean producidos en el país, y tengan como función reciclar y procesar basuras o desperdicios; y la depuración o tratamiento de aguas residuales, residuos sólidos, emisiones atmosféricas, recuperación de ríos y saneamiento básico. Ambos en el marco de un programa de mejoramiento ambiental aprobado por el Ministerio de Ambiente y Desarrollo Sostenible.

Siguiendo con esta línea, está la Ley 44 de 1990, por medio de la cual se dictan algunas disposiciones sobre catastro e impuestos sobre la propiedad raíz, que en su art. 20 establece que los municipios y departamentos podrán decretar hasta el 20\% en descuentos tributarios sobre los impuestos de vehículos que estén bajo su competencia, cuando se demuestre que cumple con las medidas para disminuir la contaminación.

Otro gran incentivo ambiental, lo reglamenta la Ley 139 de 1994, que crea el Certificado de Incentivo Forestal, y es un documento emitido por la entidad encargada del manejo y la administración de los recursos naturales renovables del medio ambiente competente, por medio del cual, se le concede el derecho a una persona beneficiaria de obtener directamente, en el momento de su presentación, por una sola vez, y en los términos que fije la entidad, las sumas de dinero de hasta: (i) el 75\% de los costos totales netos para la plantación con especies nativas; o (ii) el 50\% de los costos de plantaciones de especies introducidas; esto, siempre que se realice en densidades superiores a 1.000 árboles por hectáreas. Este incentivo tiene como principal objetivo, la reforestación en terrenos de aptitud forestal.

Este incentivo, fija unas condiciones para su otorgamiento, entre las cuales se encuentra, que sea sobre terrenos que no sean propiedad del Estado, o sobre aquellos que la normatividad ambiental obliga a conservar como bosque natural. Asimismo, la autoridad competente en otorgar este certificado debe informar a la Unidad Administrativa Especial de Parques 
Naturales cuando se vaya a realizar en áreas que hagan parte del sistema de parques nacionales.

Por su parte, el Decreto 2143 de 2015 por medio del cual se adiciona el Decreto Único Reglamentario del Sector Administrativo de Minas y Energía, establece en el art. 2.2.3.8.2.1, la deducción especial en la determinación de impuesto sobre la renta de quienes realicen de forma directa nuevas erogaciones en investigación, desarrollo en inversión en la producción y utilización de energía de hasta el 50\% del valor de las inversiones.

Paralelamente, está el Decreto 870 de 2017, que establece los parámetros para el desarrollo Pago por Servicios Ambientales y otros incentivos ambientales para la conservación y restauración, que permitan el sostenimiento y generación de servicios ambientales en zonas estratégicas. Allí se define el Pago por Servicios Ambientales como el incentivo económico dinerario o en especie dado por los interesados en servicios ambientales a los propietarios, poseedores y ocupantes de buena fe de estas áreas de preservación y restauración, a partir de acuerdos voluntarios.

Esta misma ley, enuncia los principios del Pago por Servicios Ambientales, que son:

1. Focalización, en tanto está dirigido a los propietarios, poseedores u ocupantes de buena fe, de áreas y ecosistemas reconocidos por las autoridades ambientales competentes, dando prioridad a las zonas de posconflicto.

2. Armonización, por la necesidad de la planificación, ordenamiento y gestión ambiental para reducir los daños por el uso del suelo de estas áreas, además de guiarse bajo los planes de vida de los pueblos y comunidades indígenas allí asentadas.

3. Complementariedad, en el sentido de que el Pago por Servicios Ambientales se debe realizar con conjunto con instrumentos de gestión ambiental estatales.

4. Costo efectividad, entendido como la implementación del incentivo con los recursos disponibles sobre una mayor cantidad de área, para así aumentar los resultados de conservación.

5. Posconflicto, construcción de paz y equidad, por su orientación prioritaria en áreas estratégicas de conflictos por el suelo y cultivos 
ilícitos, buscando la construcción de paz, el fortalecimiento de organizaciones campesinas y de los propietarios, poseedores $u$ ocupantes de buena de pequeña y mediana propiedad por su nivel de vulnerabilidad.

6. Solidaridad: según la cual, las instituciones públicas podrán asignar este incentivo en áreas por fuera de sus jurisdicciones, siempre que se beneficien de los servicios ambientales que estos producen.

Además de esto, se tienen unos principios adicionales para los pueblos y comunidades indígenas:

1. Territorialidad, que se encuentra definido como el territorio que estos pueblos y comunidades reconocen como esencial para su cosmovisión y existencia, incluyeron allí todos los elementos que lo complementan.

2. Autonomía y libre auto-determinación, como el ejercicio de la ley de origen o derecho propio de estos pueblos, fundados en sus cosmovisiones, que les permiten ejercer funciones jurisdiccionales, culturales, políticas y administrativas dentro de sus territorios.

Finalmente, se hará mención de la Resolución 1303 de 2018 del Ministerio de Ambiente y Desarrollo Sostenible, que modificó la Resolución 1283 de 2016 relativa al procedimiento y requisitos para la Certificación de beneficio ambiental por nuevas inversiones en proyectos de fuentes no convencionales de energía renovable y gestión eficientes de la energía, la cual permite tener beneficios tributarios. Con este cuerpo normativo, se fijan pautas para reducir el tiempo de tardanza en obtener la certificación, entre las cuales están, la eliminación del requisito de presentar copia de las autorizaciones ambientales o comunicaciones de la autoridad ambiental competente en la que conste que no es necesario ningún permiso.

De acuerdo con lo anterior, es posible afirmar que la legislación colombiana ha procurado incluir diferentes incentivos ambientales con el fin de dar cumplimiento a las disposiciones constitucionales, referida al desarrollo sostenible, hallando una armonía entre la protección del ambiente, su conservación y recuperación, y el desarrollo económico del país. 


\section{Conclusiones}

El modelo económico adoptado en la época tardía del siglo XX, basado en explotación desmedida de recursos naturales, ha generado grandes efectos en la naturaleza, se ha llegado al punto en el que la misma no puede renovarse, lo que consecuentemente afecta la supervivencia de todas especies. Es por ello que los Estados hayan comenzado a tomar medidas al respecto, creando ordenamientos jurídicos que incluían al ambiente como un derecho y con una connotación de interés público.

Colombia ha ratificado y aprobado una serie de instrumentos jurídicos internacionales relativos a la protección, conservación y recuperación del ambiente, además de ello, ha desplegado normas reforzadas de protección ambiental al estar consagrado como un derecho de todas las personas, un deber del Estado y de los particulares.

Es con base a ello que, el Estado colombiano ha establecido una serie de medidas tendientes a promover la mitigación del daño ambiental o la inexistencia del mismo, entre ellas, hallamos los incentivos ambientales, como instrumentos que buscan por medio de beneficios evitar que el actor de actividades contaminantes cause afectaciones ambientales negativas. Esto permite, que el Derecho a través del dinamismo, se mantenga a la vanguardia de los problemas políticos actuales, y tome medidas preventivas para evitar daños irreversibles.

\section{Referencias}

Amaya-Navas, Oscar, Darío (2002). La Constitución Ecología de Colombia. Análisis comparativo con el sistema constitucional latinoamericano. Bogotá: Universidad Externado de Colombia.

Caballero-Gómez, Paula (1997). Colombia y la Agenda Ambiental Internacional. Bogotá: Revista de los Andes. Recuperado de: https://revistas.uniandes.edu.co/doi/pdf/10.7440/colombiaint38.1997.02

Cruz, Gallego \& González, 2009. Sistema de Evaluación de Impacto Ambiental. Madrid: Universidad Complutense de Madrid. Recuperado de: https://eprints.ucm.es/9445/1/MemoriaEIA09.pdf

Espinoza, Guillermo (2001). Fundamentos de Evaluación de Impacto Ambiental. Santiago: Banco Interamericano De Desarrollo - BID \& Centro De Estudios Para El Desarrollo - CED. 
Levinson, Arik \& Shetty, Sudhir (1992). Efficient environmental regulation: cases studies of urban air pollution in Los Angeles, Mexico City, Cubatao, and Ankara. The World Bank: World Development Report Working Paper. Recuperado de: http://documents.worldbank.org/ curated/en/149491468781772124/pdf/multi-page.pdf

Mittermeier, Russel A., y Goettsch, Cristina. Megadiversidad. 1997. Los países biológicamente más ricos del mundo. Cemex, Ciudad de México

Organización de las Naciones Unidas (1971). Convención Relativa a los Humedales de Importancia Internacional Especialmente como Hábitat de Aves Acuáticas

Organización de las Naciones Unidas (1972). Declaración de la Conferencia de las Naciones Unidas sobre el Medio Humano.

Organización de las Naciones Unidas (1983). Convenio para la Protección y el Desarrollo del Medio Marino en la Región del Gran Caribe.

Organización de las Naciones Unidas (1983). Protocolo relativo a la Cooperación para Combatir los Derrames de Hidrocarburos en la Región del Gran Caribe.

Organización de las Naciones Unidas (1985). Convenio de Viena para la Protección de la Capa de Ozono

Organización de las Naciones Unidas (1989). Convención de Basilea sobre el control de los movimientos transfronterizos de los desechos peligrosos y su eliminación.

Organización de las Naciones Unidas (1994). Convención de las Naciones Unidas de lucha contra la Desertificación y la Sequía.

Organización de las Naciones Unidas (1992). Convenio de Brasilia sobre la Diversidad Biológica.

Organización de las Naciones Unidas (1997). Protocolo de Kioto

Organización de las Naciones Unidas (2000). Resolución 55/2: Declaración del Milenio. Asamblea General: https://undocs.org/es/A/RES/55/2

Organización de las Naciones Unidas (2002). Declaración de Johannesburgo sobre el Desarrollo Sostenible

Organización de las Naciones Unidas (2015). Acuerdo de París dentro de la Convención Marco de Naciones Unidas sobre Cambio Climático 
República de Colombia. Congreso de la República, Ley 32 de 1985 República de Colombia. Congreso de la República, Ley 44 de 1990 República de Colombia. Congreso de la República, Ley 56 de 1987 República de Colombia. Congreso de la República, Ley 74 de 1979 República de Colombia. Congreso de la República, Ley 139 de 1994 República de Colombia. Congreso de la República, Ley 165 de 1994 República de Colombia. Congreso de la República, Ley 223 de 1995 República de Colombia. Congreso de la República, Ley 253 de 1996 República de Colombia. Congreso de la República, Ley 357 de 1997 República de Colombia. Congreso de la República, Ley 461 de 1998 República de Colombia. Congreso de la República, Ley 629 del 2000 República de Colombia. Congreso de la República, Ley 788 de 2002 República de Colombia. Congreso de la República, Ley 1450 de 2011 República de Colombia. Congreso de la República, Ley 1536 de 2012, República de Colombia. Congreso de la República, Ley 1819 de 2016 República de Colombia. Corte Constitucional, Sentencia C 644 del 18 de octubre del 2017.

República de Colombia. Ministerio de Ambiente y Desarrollo Sostenible. Decreto 870 de 2017

República de Colombia. Ministerio de Ambiente y Desarrollo Sostenible. Decreto 2143 de 2015

República de Colombia. Ministerio de Ambiente y Desarrollo Sostenible. Resolución 1283 de 2016

República de Colombia. Ministerio de Ambiente y Desarrollo Sostenible. Resolución 1303 de 2018

Reyes, Mauro \& Vargas, Andrés (2010). Incentivos económicos para la conservación de áreas naturales: Una revisión de la literatura. Medellín: Universidad de Antioquia. Recuperado de: http://aprendeenlinea.udea.edu.co/revistas/index.php/lecturasdeeconomia/article/ view/9997/17987

Tratado de Cooperación Amazónica de Brasilia. (1978) 
Los incentivos como política para favorecer las innovaciones ambientales

Van Hecken, Gert. \& Bastiaensen, Johan (2010). Payments for Ecosystem Services in Nicaragua: Do Marketbased Approaches Work? Development and Change, 41(3): 421-444. 